\title{
The Rotor and Tower Vibrations Damping Monitoring in the Case of Total Pitch Servomotors Failure
}

\author{
Younes Ait Elmaati, Lhoussain El Bahir and Khalid Faitah \\ Department of Electrical Engineering and Control, National School of Applied Sciences, Cady Ayad University, \\ Postal:575, Abdelkarim Khattabi Avenue, Gueliz, Marrakech, Morocco.
}

(Received 3 July 2018; accepted 22 November 2018)

\begin{abstract}
In this paper, a strategy is proposed to deal with the total pitch actuator failure in the modern wind turbines. The fault is firstly detected using a suitable proportional residual which rises when the measured rotor dynamics match the pure wind turbulence driven dynamics of the reference model. The fault tolerant control method relies on the distribution of the control signal on the remaining actuators, namely the generator torque and the yaw actuator. The pseudo inverse is applied on the residual between the measured and the nominal dynamics of the tower and the rotor. The distributed control signal is then suitably filtered in order to match the production and the safety objectives. Firstly, the generator torque filter comes to minimize the high frequencies on the rotor speed for a stable production to the grid. Secondly, the band pass yaw angle filter comes to minimize the mean yaw misalignment between the yaw nacelle and the wind direction. The method shows good dynamics reconstruction capabilities while keeping a stable production within a safe environment.
\end{abstract}

\section{INTRODUCTION}

\subsection{The Nominal Production of the Wind Turbine}

The wind turbine is a device to extract the energy contained in the wind through a succession of components. The first component is the rotor, containing two, three or more blades. The three bladed wind turbines are the most popular due to their high efficiency. The three blades are spaced with $120^{\circ}$ in order to achieve a symmetry useful in the cancelation of the loads on the wind structure. The loads alleviation is performed by actuating three servomotors in the blades, rotating about the longitudinal axis of the blades by a pitch angle $\beta$. This operation allows facing the WT to the wind $\left(\beta=0^{\circ}\right)$ or not $\left(\beta=90^{\circ}\right)$. The second component is the tower; it is responsible of carrying the nacelle to a suitable wind level. It is also responsible of routing the wirings from the generator to the transformer in the foundation. The tower experiences a lot of vibrating movement (side to side and fore aft) due to the high turbulence of the wind, especially for large structures ( $>40 \mathrm{~m}$ ). The pitch actuators are usually used to damp those movements through suitable control strategies. ${ }^{1-3}$

In the wind turbines, there are two other actuators, namely the generator and the nacelle yaw. The generator electromagnetic torque is used to accelerate or decelerate the wind turbine in the region of the moderate winds $(>3 \mathrm{~m} / \mathrm{s}$ and $<7 \mathrm{~m} / \mathrm{s})$. The objective is to maximize the amount of caught energy. ${ }^{4,5}$ The principle is to act on the rotor speed in order to maintain the power coefficient at its optimal value. The produced moment by the generator torque belongs to the rotation plan $\left(y_{n}, z_{n}\right)$ in Fig. 1. In the region of the so-called high winds, the generator torque is used to damp some vibrations in the plan of rotation such as the torsional moment on the principle shaft of the WT.

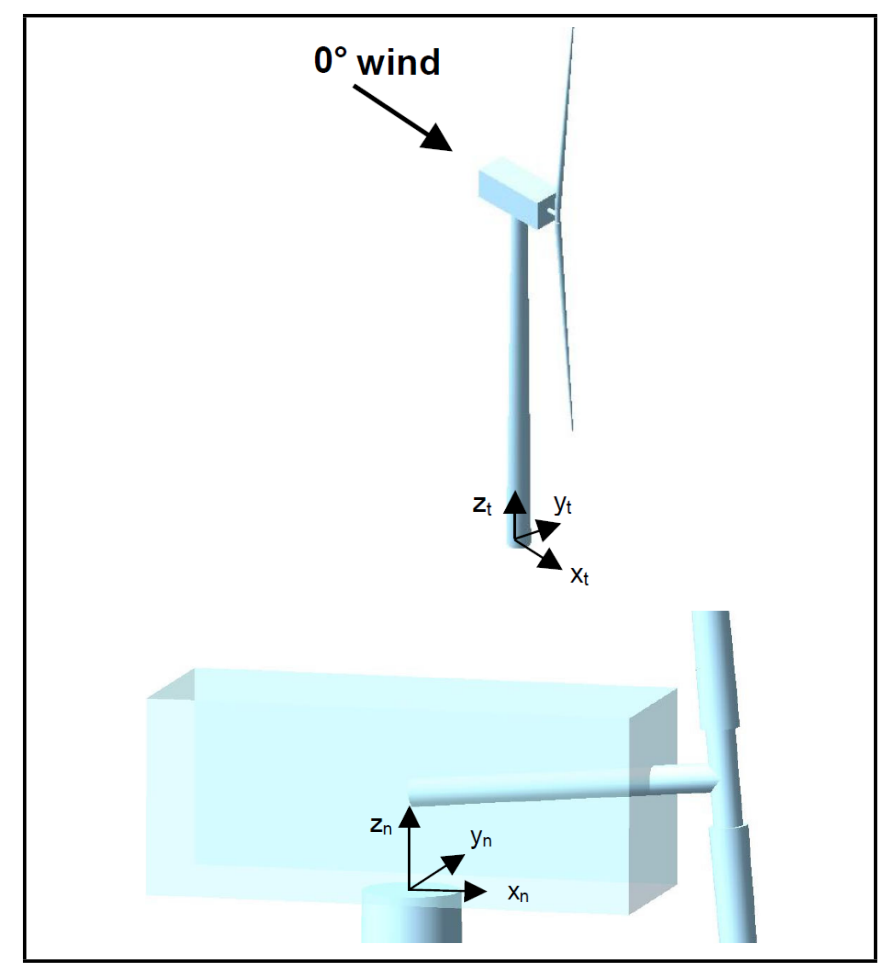

Figure 1. The tower (top) and the nacelle (bottom) axis.

The third actuator is the nacelle yaw (Fig. 2). It is composed of three electric motors and gears allowing a rotation in the $\left(x_{n}, y_{n}\right)$ plan in Fig. 1 . The electrical motors are driven by DC voltage to rotate at a yaw angle $v$ with a yaw rate $\dot{v}$. This rotation allows increasing the energy capture through pointing the rotor swept area steadily towards the incoming wind direction and thus maximize the general power input of the wind turbine. Moreover, the yaw actuator allows the reduction of the structure load by letting the nacelle rotate to compensate 


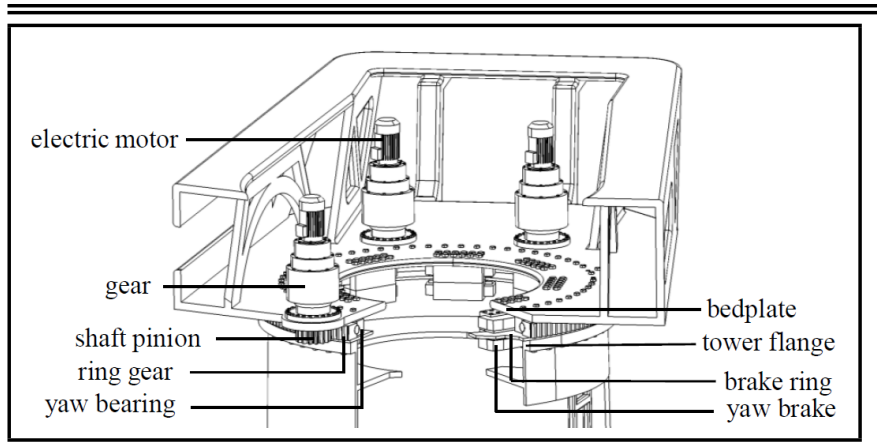

Figure 2. The yaw actuator mechanical component.

the aerodynamic loads. The constraint on this last actuator is its limited bandwidth, which at most achieves $10^{\circ} /$ s. $^{6,7}$ This actuator, when driven at a mean yaw angle different from the wind direction, induces a phenomenon of yaw misalignment. The last phenomenon leads to create yaw loads on the nacelle structure. Generally, a yaw misalignment of $\pm 8^{\circ}$ must be considered if the very small angles could not be achieved.

The pitch servomotors previously discussed are, like all the industrial actuators, exposed to failures for different reasons. Namely, the usury of the mechanical shafts, electrical circuits especially the power supply. For this reason, a nominal study is insufficient and the dysfunctional study becomes mandatory.

\subsection{The Faults and Fault Tolerant Control in the Wind Turbines}

\subsubsection{Preliminaries}

The first step in the fault tolerant control is the fault detection and isolation (FDI). The most relevant FDI methods are based on residuals. In residual-based FDI, signals from a mathematical model and hardware measurements are compared and the filtered difference forms a residual signal. ${ }^{8}$ In nominal fault-free conditions, the residuals should be zero, and nonzero when faults/failures occur. This residual signal is usually applied with a threshold to avoid false alarms from disturbances or uncertainty. When the residual signal exceeds the threshold, a fault is said to occur. The second step is the design of the fault tolerant controller (FTC) which could be passive or active. Passive FTC is usually based on robust control ideas and therefore handles faults/failures without requiring information from an FDI scheme. ${ }^{9}$ Active FTC (AFTC) in general requires some information on the faults/failures that occur and therefore typically FDI is required. ${ }^{10}$ The paper Precup et al., presents an overview of the fault diagnosis methods categories with an application on the industrial pitch wind turbines. ${ }^{11}$

\subsubsection{The case of the industrial modern wind tur- bines}

In the field of wind turbines, it is shown as in Ribrant and Bertling that gearbox faults are the most critical ones, essentially due to its relatively high stopping time and severity on the whole structure. ${ }^{12,13}$ In the second rank one find electrical faults such as short circuits, over voltage of the inverter, hard over for the generator and sensor faults like drift or bias. Another essential component is the anemometer. The anemometers are used in the feedforward control of the wind turbines.
In Elmaati et al., authors proposed a new method to estimate the wind speed and replace the anemometers. ${ }^{14}$ The strength of the method is to avoid numerical solutions used in literature of the wind speed estimation. In fact, it uses a simple integrator to extract the wind speed instead of complex mathematical tools. The most popular papers in the field, deal with the faults in the pitch servomotors actuators due to their impact on the structure. For example, Rezaei and Johnson considers the leakage in the hydraulic liquid in which the servomotors exist. $^{15,16}$ The signature of this fault is a decrease in the dynamics of the servomotors. Other papers have considered the pitch actuators loss of efficiency. ${ }^{17}$ It is a fault where the actuator gives a limited response when solicited by the controller. This fault impacts the loads on the wind turbine, because the delivered action is not sufficient to achieve the desired load cancelation level. ${ }^{18,19}$ The same effect could be produced by a fault on the load sensors. In fact, an additive bias on the blades moment sensor leads to an additive action from the pitch actuators and hence an over actuation. In contrast, a subtractive bias on the tower sensor will lead to an insufficient control action and then the loads are not completely canceled. Authors in Wei and Verhaegen (2011) proposed a robust observer to generate residuals for the fault turbine moment sensors which provides a measurement of the loads applied on the structure..$^{20}$

\subsubsection{The main contribution of the paper}

The majority of the papers in the wind turbine fault tolerant control field considers only partial faults on the pitch actuators. However, the total pitch actuator has not been considered before. This fault could occur when the power supply to the pitch actuators is cut off. Up to date, the available solution is only to shut down the wind turbine and wait for the curative maintenance intervention. This induces a relatively long shutdown time, and a high energy production cost.

For the previous reasons, the present paper proposes a strategy to firstly detect, then control the wind turbine in the presence of total pitch actuators fault. The contributions are as follows:

1. The proposition of a strategy to detect the total actuator failure based on the measured dynamics and the estimated wind dynamics.

2. The proposition of a filtered redistributed control signal for the reconstruction of the pre-fault dynamics while minimizing the mean yaw misalignment from the wind direction.

3. The validation of the strategy on a multi objective benchmark control case study with respect to industrial limits on the yaw rate and yaw misalignment.

This paper is partitioned into four sections. Section 1 gives some preliminaries and state of art of the fault tolerant control in wind turbines. After that, the wind turbine model is derived, and the used software is presented in section 2 . In the same section, the linear quadratic control of the wind turbine is performed. Section 3 presents the contribution on the fault detection and the fault tolerant control in the total failure of pitch control. Finally, the paper will be concluded with section 4 . 


\begin{tabular}{l}
\hline \hline Table 1. The considered wind turbine characteristics. \\
$\qquad$\begin{tabular}{|l|l|}
\hline Characteristic & Value \\
\hline Rated power $(\mathrm{MW})$ & 1.5 \\
Rotor diameter $(\mathrm{m})$ & 70 \\
Blade mass $(\mathrm{kg})$ & 4,230 \\
Hub mass $(\mathrm{kg})$ & 15,104 \\
Total rotor mass $(\mathrm{kg})$ & 32,016 \\
$K_{\text {Tower }}:$ Stifness of tower $(\mathrm{N} \cdot \mathrm{m} / \mathrm{rad})$ & $6.4643^{*} 10^{5}$ \\
$D_{\text {Tower }}$ Damping of the tower $(\mathrm{N} \cdot \mathrm{m} / \mathrm{rad} / \mathrm{s})$ & 45369 \\
Jr: Rotor Inertia $\left(\mathrm{kg} \cdot \mathrm{m}^{2}\right)$ & $2.9624 * 10^{6}$ \\
Jg: Generator Inertia $\left(\mathrm{kg} \cdot \mathrm{m}^{2}\right)$ & $53.036^{*} \mathrm{Ng}^{2}$ \\
Ng: Gearbox Ratio & 87.965 \\
$\mathrm{R}:$ Rotor radius $(\mathrm{m})$ & 35 \\
$M_{t}:$ Tower mass $(\mathrm{Kg})$ & 98850 \\
\hline
\end{tabular}
\end{tabular}

\section{THE WIND TURBINE MODEL}

\subsection{The FAST Software (Fatigue, Aerodynamics, Structure and Turbulence)}

The FAST (Fatigue, Aerodynamics, Structures, and Turbulence) code is an aeroelastic simulator capable of predicting both the extreme and fatigue loads of two- and three-bladed flexible horizontal-axis wind turbines (HAWTs) with 24 degrees of freedom. ${ }^{21,22}$ The code uses a modal approach in combination with Kane Dynamics to develop the equations of motion. FAST helps researchers and engineers with testing wind loads and reduction algorithms. ${ }^{23}$ Another relevant field of research is testing the effect of faults and fault tolerant control strategies on the different loads of the turbine. The last test determines the suitable algorithm to use in order to avoid the excitation of the turbine vibrational modes.

\subsection{The Wind Turbine Model}

The considered degrees of freedom were the rotor rotation in the $\left(y_{n}, z_{n}\right)$ plan and the tower fore aft displacement in the nonrotating plan $\left(z_{t}, x_{t}\right)$. The considered operating point was $40 \mathrm{rpm}$ of rotor speed, $12.73 \mathrm{KNm}$ of generator torque, $0^{\circ}$ of yaw angle and $9.0093^{\circ}$ of pitch angle. The wind turbine was driven by the aerodynamic torque of the wind defined by Eq. (1):

$$
T_{a}=\frac{1}{2} \sigma \pi R^{3} V^{2} C_{q}\left(V, \Omega_{r}, \beta\right) .
$$

The parameter $\sigma$ represented the air density and was equal to $1.125 \mathrm{Kg} / \mathrm{m}, R$ was the radius of the rotor, $V$ was the wind speed, $C_{q}$ represented the amount of torque extracted from the available torque of the wind, $\Omega_{r}$ was the rotor speed and $\beta$ was the pitch angle. $C_{q}$ was usually given by the constructor for each wind turbine as data point.

After the application of the first law of mechanics (Newton) on the rotor and the tower, the extracted model is given by:

$$
\begin{array}{r}
\left(\begin{array}{c}
\delta \dot{x}_{\mathrm{TFA}} \\
\delta \ddot{x}_{\mathrm{TFA}} \\
\delta \dot{\Omega}_{r}
\end{array}\right)=\left[\begin{array}{ccc}
0 & 1 & 0 \\
-\frac{K_{\text {Tower }}}{M_{t}} & -\frac{D_{\text {Tower }}}{M_{t}} & 0 \\
0 & 0 & \frac{\gamma}{J_{t}}
\end{array}\right]\left(\begin{array}{c}
\delta x_{\mathrm{TFA}} \\
\delta \dot{x}_{\mathrm{TFA}} \\
\delta \Omega_{r}
\end{array}\right) \\
+\left[\begin{array}{c}
0 \\
\frac{F_{p}}{M_{t}} \\
\frac{\rho}{J_{t}}
\end{array}\right]\left(\begin{array}{l}
\delta \beta_{1} \\
\delta \beta_{2} \\
\delta \beta_{3}
\end{array}\right)+\left[\begin{array}{c}
0 \\
\frac{d}{M_{t}} \\
\frac{\alpha}{J_{t}}
\end{array}\right] \delta \omega ;
\end{array}
$$

where $F_{p}=-3.1473 .10^{6} ; d=40171$. The considered turbine was a Wind PACT 1.5 MW large scale. More details about the parameters of this wind turbine could be found in Poore and Lettenmaier and Perfiliev et al. ${ }^{24,25}$ The turbine parameters summary are given in Table 1 . The operator $\delta$ was used to indicate the variations about the operating point. The model was a state space representation given by:

$$
\delta \dot{x}=A \delta x+B_{1} \delta u+D_{1} \delta \omega
$$

where:

$$
\begin{gathered}
A=\left[\begin{array}{ccc}
0 & 1 & 0 \\
-\frac{K_{\text {Tower }}}{M_{t}} & -\frac{D_{\text {Tower }}}{M_{t}} & 0 \\
0 & 0 & \frac{\gamma}{J_{t}}
\end{array}\right] ; B_{1}=\left[\begin{array}{c}
0 \\
\frac{F_{p}}{M_{t}} \\
\frac{\rho}{J_{t}}
\end{array}\right] ; \\
D_{1}=\left[\begin{array}{c}
0 \\
\frac{d}{M_{t}} \\
\frac{\alpha}{J_{t}}
\end{array}\right] ; \quad \delta x=\left(\begin{array}{c}
\delta x_{\mathrm{TFA}} \\
\delta \dot{x}_{\mathrm{TFA}} \\
\delta \Omega_{r}
\end{array}\right) ; \quad \delta u=\delta \beta ;
\end{gathered}
$$

with $\delta \beta=\delta \beta_{1}=\delta \beta_{2}=\delta \beta_{3} . \quad \delta \beta_{i}$ was the control signal delivered to the servomotor of index $i$. $A$ was the dynamics matrix; $B_{1}$ was the baseline input matrix; $D_{1}$ was the disturbance input matrix. $\delta x$ was the state vector; $\delta u$ was the pitch input vector; $\delta \omega$ was the disturbance input vector. The parameters $d$ and $\alpha$ were respectively the effect of the wind disturbance on the tower and the rotor speed.

It was assumed that all the three states are measurable. In fact, a rotor speed sensor and an accelerometer were usually physically available to measure the speed and the tower fore aft (TFA) gradient in meters/s. The tower fore aft displacement in meter could be obtained by integrating the measured TFA gradient. The $C$ matrix was then identified as $I_{3}$. The values of the model parameters are as follows:

$$
\begin{gathered}
\gamma=\frac{\partial T_{a}}{\partial \Omega_{r}}=-0.1039 J_{t} ; \\
\rho=\frac{\partial T_{a}}{\partial \beta}=-2.5727 J_{t} ; \\
\alpha=\frac{\partial T_{a}}{\partial V}=0.061141 J_{t} ;
\end{gathered}
$$

where $J_{t}=J_{r}+J_{g} N_{g}^{2}$ was the total inertia of the wind turbine shaft.

\section{THE BASELINE CONTROLLER DESIGN}

\subsection{Rotor Speed and Tower Fore-Aft Control Using the Pitch Angle Linear Quadratic Regulator}

The control of the wind turbine was multi objective. Namely, the control of the rotor speed while damping the tower structure vibrations. The regulation was performed through a Linear Quadratic controller (LQ). The choice of this controller comes in the aim that the pitch control efforts are to be optimized in order to match the industrial accepted ranges. The objective was to find the state feedback control law $u=-\mathrm{Kx}$ that minimizes the cost function:

$$
J=\int x^{\mathrm{T}} Q x+u^{\mathrm{T}} R u \mathrm{~d} t .
$$


Subject to the system dynamics $\dot{x}=A x+B u$.

$Q$ and $R$ were the weighting matrices used to optimize the control signal and or the system states. In the present case, the values of those matrices were fixed to:

$$
Q=\left[\begin{array}{ccc}
1 & 0 & 0 \\
0 & 10 & 0 \\
0 & 0 & 7
\end{array}\right] ; \quad R=20
$$

$R=20$ was chosen to promote the minimization of the pitch angle control signal. $Q(1,1)$ was chosen superior to $Q(3,3)$ in order to promote the tower fore aft variations optimization in front of rotor speed variations. The optimal control law is given by:

$$
K=R^{-1} B^{T} P
$$

where $P$ was a real, positive semi definite matrix, solution of the Riccati equation:

$$
A^{T} P+P A-P B R^{-1} B^{T} P+Q=0 .
$$

The resulting control vector is $\left(\begin{array}{lll}-0.4159 & -0.6765 & -0.4684\end{array}\right)$. The initial eigenvalues of the dynamic matrix were composed of two conjugates representing the tower vibrations which are $-0.2295+2.5472 \mathrm{i}$ and $-0.2295-2.5472 \mathrm{i}$; and another real eigenvalue representing the rotor speed -0.1039 . The objective of the control was to damp the oscillations by making the eigenvalue of the tower negative real. The natural frequency of the tower equals $0.4 \mathrm{~Hz}$ and its expression is given by:

$$
f_{\mathrm{n}_{-} \text {Tower }}=\sqrt{\frac{\text { Damping Coefficient }}{\text { Tower Mass }}}=\sqrt{\frac{45369}{98830}}=0.4 \mathrm{~Hz} \text {. }
$$

In the sequel, this frequency was used to design the filter on the redistributed signal in the faulty case. After applying the resulting controller $K$, the eigenvalues of the closed loop dynamic matrix became $(-23.0438-0.4351 \pm 0.0004 i)^{T}$. The imaginary part could be neglected since it was sufficiently inferior to the real part. So the resulting new poles of the LQR regulated system give the following second order equation:

$$
\begin{gathered}
(s+23.0438)(s+0.4351)=s^{2}+23.4789 s+10.0264 . \\
(s+23.0438)(s+0.4351)=s^{2}+2 \theta \omega_{n} s+\omega_{n}^{2}
\end{gathered}
$$

The parameter $\theta$ was called the damping ratio. By the identification of the same order terms between in Eq. (11) and Eq. (12), the closed loop damping ratio $\theta$ was equal to 3.7075 , which was sufficiently superior to 1 . Let's define the performance of the oscillations damping function of $\theta$ as:

$$
\text { Perf }=1-D_{\%}
$$

where the variable $D_{\%}$ is the overshoot of the rotor speed and tower fore aft displacement from the operating point. The expression of $D_{\%}$ was given in Shamsuzzoha and Skogestad as: ${ }^{26}$

$$
D_{\%}=100 \exp \left(\frac{-\theta \pi}{\sqrt{1-\theta^{2}}}\right) .
$$

The Fig. 3 illustrates the evolution of the damping performance and the overshoot with respect to the damping ratio $\theta$.

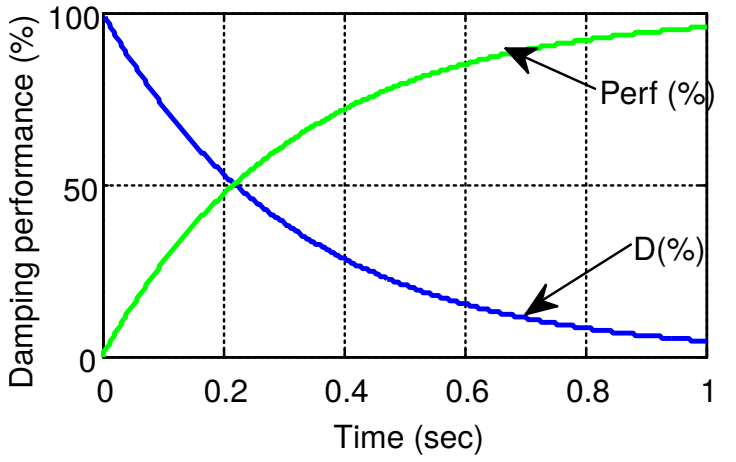

Figure 3. The damping performance evolution function of the damping ratio $\theta(\%)$.

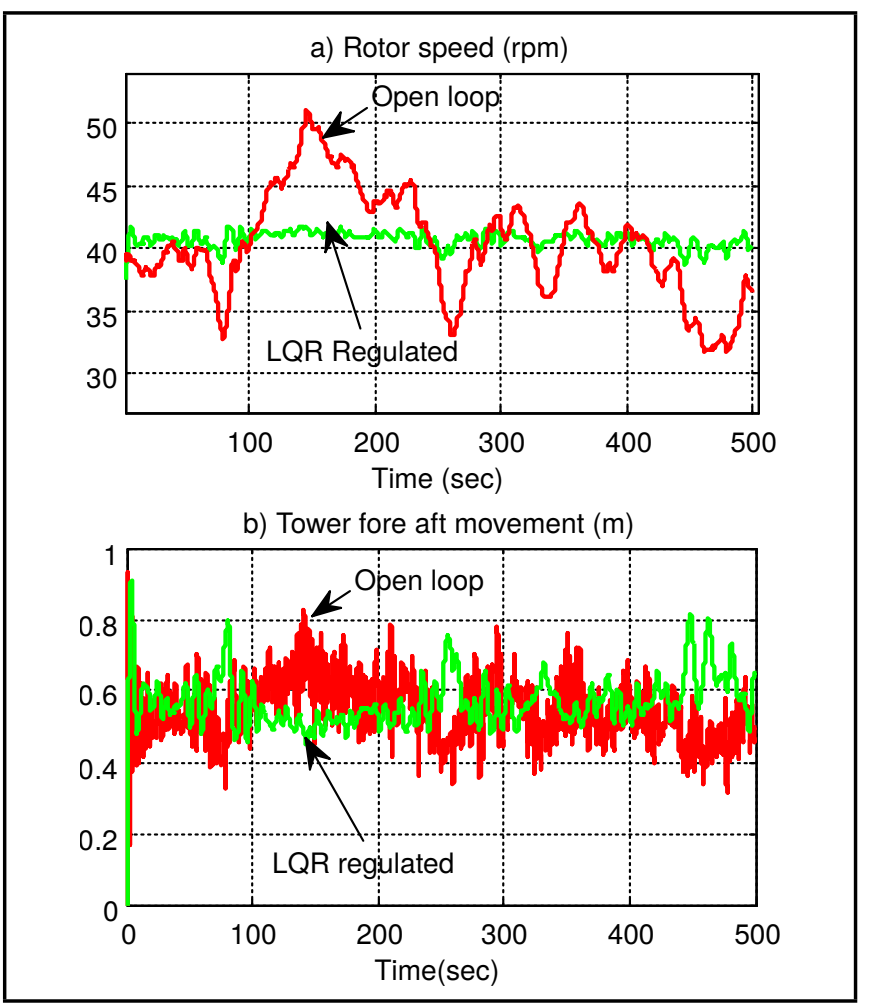

Figure 4. The rotor speed (a) and the Tower fore aft displacement (b).

For values of the damping ratio $\theta$ near 1 , the performance of damping is maximal. The system was called critically damped since $\theta=1$. This damping level allowed a maximum diminution in tower movement amplitude to $95 \%$ compared to the open loop performances.

The objective of the regulation was to minimize the overshoot $D$ in order to minimize the loads on the rotor and the tower. The last fact means that it was expected to obtain rotor speed and tower fore aft overshoot $D$ at most $5 \%$ in the closed loop. From Fig. 4a, at 150s, a regulated rotor speed of $42 \mathrm{rpm}$ with an overshoot of $2 \mathrm{rpm}$ from the operating point $40 \mathrm{rpm}$ which means $2 / 40=0.05=5 \%$.

\subsubsection{The $L Q$ regulation results}

The set of figures from Fig. 4 to Fig. 7 illustrates the results of the nominal regulation of the rotor speed and the tower fore aft movement using the LQ regulator. 


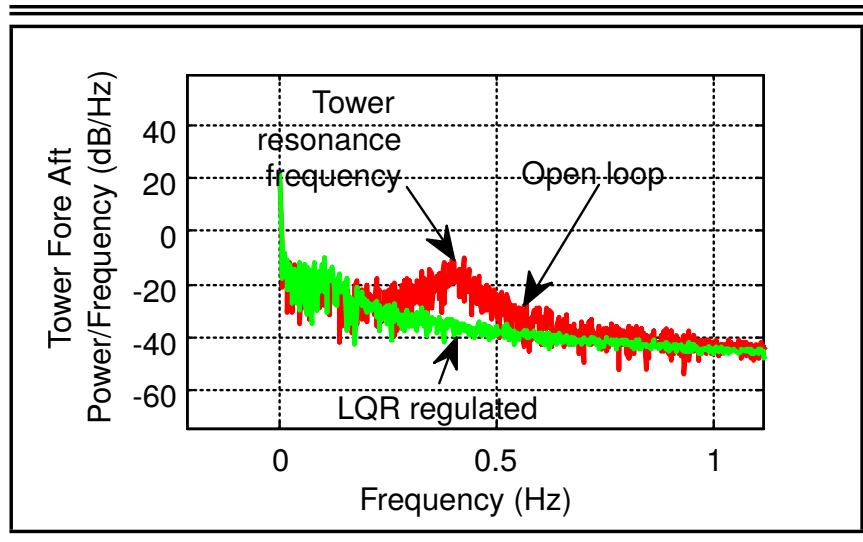

Figure 5. The tower fore aft displacement frequency content $(\mathrm{dB} / \mathrm{Hz})$.

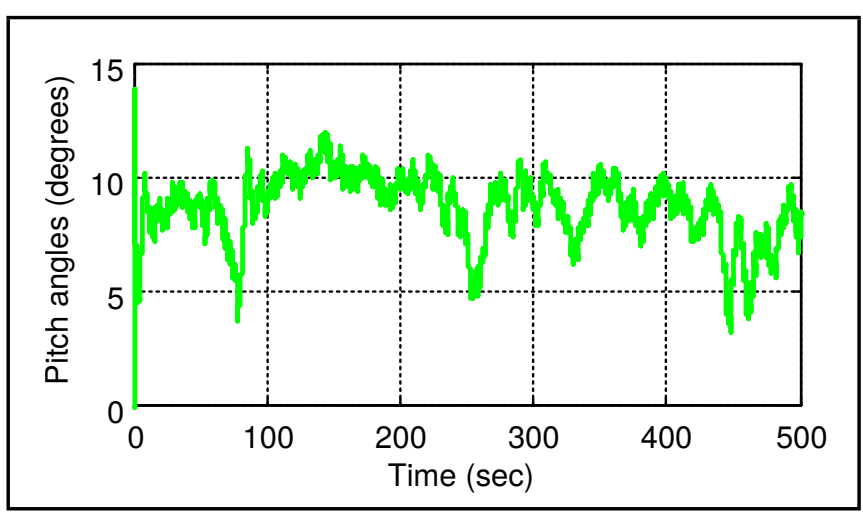

Figure 6. The blades pitch angle (Degrees).

As expected earlier in the paper, the rotor speed and the tower fore aft are damped with at most $5 \%$ of overshoot from the operating point. In fact, the rotor speed overpassing equals $\frac{42-40}{40}=5 \%$, and the tower fore aft equals $\frac{0.55-0.58}{0.58}=2 \%$.

Figure 5 shows the Power/Frequency representation of the tower fore aft before and after LQ regulation. At $0.4 \mathrm{~Hz}$, one can notice a resonant behavior of the tower in the open loop. This frequency was canceled with the closed loop regulation.

Figure 6 shows the pitch angles evolutions with respect to the tower fore aft and rotor speed variation due to the wind. The pitch angle control signal is composed of two parts. The first part was low frequency (less than $0.2 \mathrm{~Hz}$ ) used by the pitch servomotors to regulate the rotor speed. Between the instants $100 \mathrm{~s}$ and $250 \mathrm{~s}$, the rotor speed becomes larger than the operating points $40 \mathrm{rpm}$. For instance, in the $150 \mathrm{~s}$ instant, it becomes $49 \mathrm{rpm}$, which means a $9 \mathrm{rpm}$ of increase. For this reason, the controller increased the pitch angle with 3.5 degrees to attenuate the $10 \mathrm{rpm}$. In fact, $10 \mathrm{rpm}$ is equal to $3.5 * 2.5727=$ $9.0045 \mathrm{rpm}$.

The second part of the control signal was high frequency $(0.4 \mathrm{~Hz})$ used to regulate the tower fore aft movement (Fig. 7). Between the instants $135 \mathrm{~s}$ and $136 \mathrm{~s}$, an increase of $0.25 \mathrm{me}-$ ters induces an increase of the pitch angles of 0.65 degrees, which is equal to $0.25 * 2.5727=0.6432$. Where 2.5727 corresponded to the pitch angles input matrix to the tower fore aft movements. a) Tower fore aft movement (m)

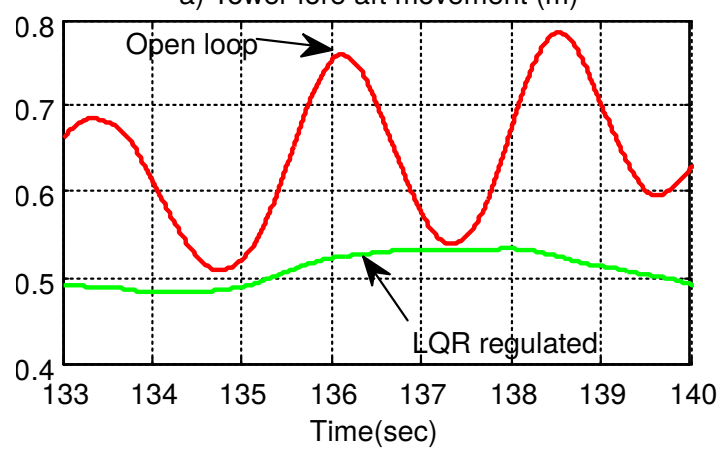

b) Pitch angles (degrees)

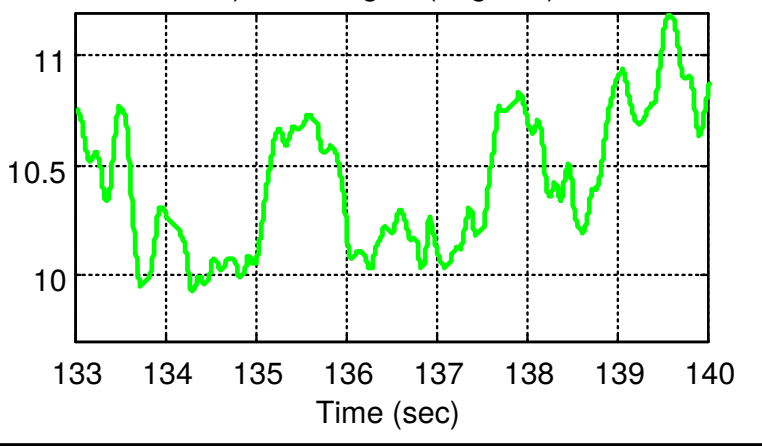

Figure 7. The tower fore aft (a) and the pitch angle (b) in the zone [134;140] s.

\section{THE TOTAL PITCH ACTUATOR FAULT}

\subsection{The Problem Statement}

The faulty case is the total failure of the pitch actuators. This fault makes the system behaves as an open loop, loosing completely the regulation performance. The problem solution can be formulated as follows:

Find a new control law $u_{f}$ so that

- The dynamics $\{\text { Rotor, Tower }\}_{\text {faulty }}$ $\underset{t \rightarrow \infty}{\longrightarrow}\{\text { Rotor, Tower }\}_{\text {reference }}$

- The low frequency mean yaw misalignments are canceled

Firstly, a fault detection bloc should be designed to activate the strategy when the total fault of pitch actuators occurs. This bloc is derived from a reference model states equation as follow:

$$
C \dot{x}_{m}(t)=C A x_{m}(t)+C B u(t)+C D_{1} \delta \omega(t) .
$$

$x_{m}(t)$ is the reference model state vector; $\delta \omega(t)$ is the wind disturbance about the operating wind of $18 \mathrm{~m} / \mathrm{s}$. The matrices $A, B, C$ and $D_{1}$ are respectively the dynamics, the input action, the measurements and the disturbance action matrices and are known.

In the total pitch failure, the input action becomes $B=0$ (NULL matrix). The model reference dynamics becomes:

$$
C \dot{x}_{m}(t)=C A x_{m}(t)+C D_{1} \delta \omega(t) .
$$

If the fault occurs, the actual measured dynamics $\delta \dot{y}(t)=$ $C \dot{x}(t)$ match the pure wind driven model reference dynamics 
$C \dot{x}_{m}(t)$ (equivalent to open loop dynamics). If no fault occurs, the relationship between the measured dynamics $\delta \dot{y}(t)$ and the reference dynamics $C \dot{x}_{m}(t)$ obey to the baseline controller damping specifications. This means that in the normal case, if a tower damping level of $90 \%$ is specified by the baseline controller, the measured dynamics will represent $10 \%$ of the pure wind driven reference dynamics. In the faulty case, the measured dynamics will represent up to $100 \%$ of the wind driven dynamics, which means that all the dynamics will be driven by the wind and no dynamics will be controlled by the baseline controller.

\subsection{The Fault Detection Bloc}

The signature of the pitch actuator total failure is that the actual dynamics (measured) and the open loop dynamics (wind driven reference dynamics) matches.

From all the explanations above, the detection residual could be defined as follows:

$$
\epsilon(\%)=100 \times \frac{\delta \dot{y}(t)}{C A x_{m}+C D_{1} \delta \omega(t)} .
$$

The turbulence variations $\delta \omega(t)$ about the operating point could be obtained using anemometers. The last devices are known with their inaccurate measurement and are exposed to faults. The last fact could lead to false alarms and then wasted reconfiguration efforts. For this reason, the estimation $\delta \hat{\omega}(t)$ of the turbulence is used as in Elmaati et al. ${ }^{14}$ In this reference, the expression of the square estimated wind speed is given by the turbine depending transfer function:

$$
\delta \hat{\omega}^{2}=\frac{\tau}{\tau s+1} \times \frac{1}{s} \times \frac{a s+b}{s^{2}+c s+d} \times \delta \Omega_{r} ;
$$

where $s$ is the Laplace operator and $\delta \omega_{r}$ is the rotor speed. The parameters $a, b, c$ and $d$ are wind turbine dependent constant coefficients. The coefficient $\tau$ is a design parameter to choose the bandwidth of the wind frequencies to estimate. The fault occurrence hypothesis is then as follow:

$$
\begin{aligned}
& H_{0}: \epsilon \leq 10 \% \text { No total pitch actuator fault } \\
& H_{1}: \epsilon \simeq 100 \% \text { pitch actuator total failure }
\end{aligned}
$$

The threshold of $10 \%$ corresponds to the damping performances specified in the baseline controller. The advantage of the residual proposed in this paper is that the threshold for the no total fault hypothesis is easily known in contrast to the traditional subtractive residual. In the latter case, the fault decision threshold is not easily found. Figure $8 \mathrm{a}$ illustrates the rotor speed dynamics before and after the fault occurrence in the instant $150 \mathrm{~s}$. The dynamics in the interval $[0 ; 150] \mathrm{s}$ matches the healthy regulated dynamics about the operating point ( $95 \%$ of rotor speed damping).

Above the instant of $150 \mathrm{~s}([250,450] \mathrm{sec}$ for example), the fault could be detected as the rotor measured dynamics $\delta \dot{\Omega}_{r}(t)$ (blue) matches the pure wind driven rotor reference dynamics $\delta \dot{\Omega}_{\mathrm{rm}}(t)$ (green) after some transitory due to non-modeled modes of the structure (blades...) and the open loop. The pitch actuators total failure is detected when the measured dynamics

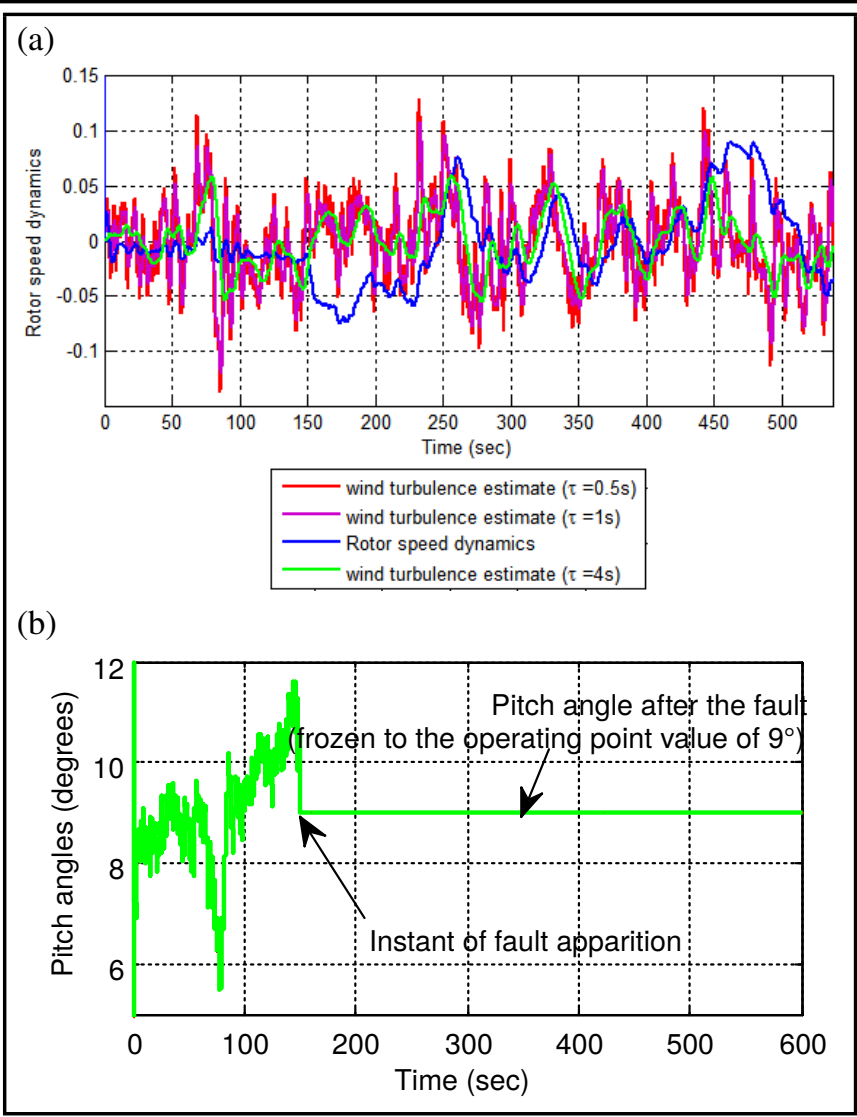

Figure 8. The rotor speed dynamics and the wind turbulence estimated dynamics.

and the reference dynamics driven only by the wind matches. In fact, in the fault free situation, the dynamics of the wind turbine are driven by two vectors: the control vector $\mathrm{u}$ and the wind $\delta \omega(t)$. In the faulty situation, the control term $\mathrm{Bu}(\mathrm{t})$ disappear because $\mathrm{B}=0$ (null matrix) and the dynamics are driven only by the wind turbulence. If the residual is less than $100 \%$ and more than $10 \%$, it could be interpreted as a partial pitch fault. In this case, the input matrix $B$ is not null but multiplied by a fault coefficient $\kappa$ such as $0<\kappa<1$. This case is not considered in this paper.

The Fig. $8 \mathrm{~b}$ represents the pitch angles. In the interval $[0,150] \mathrm{s}$, the pitch angles obey to the control law delivered by the baseline controller. Above 150s, the pitch angles are frozen to the operating value of $9^{\circ}$.

\subsection{The Fault Tolerant Control Strategy}

The solution to the problem in the paragraph 4.1 is to distribute the control signal on the remaining healthy actuators, which are the generator and the yaw actuator. The new input matrix of the healthy actuators is given by:

$$
B_{2}=\left(\begin{array}{c}
\frac{F_{y r}}{M_{t}} \\
\frac{F_{g}}{J_{t}}
\end{array}\right)=\left(\begin{array}{cc}
0 & 0 \\
\frac{F_{g 1}}{J_{t}} & \frac{F_{y r 1}}{M_{t}} \\
\frac{F_{g 2}}{J_{t}} & \frac{F_{y r 2}}{J_{t}}
\end{array}\right) .
$$

The vector of the new inputs is:

$$
u_{f}=\left[\begin{array}{c}
\delta T_{g} \\
\delta \dot{\theta}_{r}
\end{array}\right] .
$$


The scalars $F_{g 1}$ and $F_{y r 1}$ are respectively the generator and yaw rate effect on the tower fore aft gradient. $F_{g 2}$ and $F_{y r 2}$ are respectively the generator and yaw rate effect on the rotor speed. Where $F_{g 1}=4.375 .10^{-5} J_{t} ; F_{g 2}=-175 ; F_{y r 1}=$ $-2.8292 .10^{5} ; F_{y r 2}=49345$.

The fault tolerant control signal is constructed by inverting the new input matrix $B_{2}$. This inversion is applied to the residual between the reference dynamic $\dot{Y}_{m}$ and the actual dynamic of the faulty system $\dot{Y}_{f}$. Let's call $r=\dot{Y}_{m}-\dot{Y}_{f}$ this residual. It is given by:

$$
\begin{gathered}
\dot{Y}_{f}=C A \delta x+C B_{2} u_{f}+C D_{1} \delta \omega . \\
\dot{Y}_{m}=C A \delta x_{m}-B K \delta x . \\
r(t)=C A \delta x_{m}-B K \delta x-C A \delta x-C B_{2} u_{f}-C D_{1} \delta \omega .
\end{gathered}
$$

$\delta x_{m}$ is the vector of the reference generated model states, $\delta x$ is the vector of the measured system states, $u_{f}$ is the new control law to find. $K$ is the control matrix designed previously using the LQ regulator.

The new control law can be derived such as the residual between the reference dynamic $\dot{Y}_{m}$ and the actual dynamic of the faulty system is canceled. The cancelation of the residual necessitates the new control law $u_{f}$ to be:

$$
u_{f}=\left(C B_{2}\right)^{+}\left(C A \delta x_{m}-B K \delta x-C A \delta x-C D_{1} \delta \omega\right) .
$$

\subsubsection{The control signal after treatment}

Firstly, a band pass filtering of the yaw angle about the tower natural frequency should be performed. Then, the new yaw rate is obtained by filtering the yaw angle as:

$$
\dot{\theta}_{f}=\frac{s}{\mu s+1} \theta_{f}
$$

where $\mu$ is the time constant and should be sufficiently high to approximate a derivative.

The band to be passed through the yaw angle should match an interval about the natural frequency of the tower $0.4 \mathrm{~Hz}$. This interval is $(0.4-0.13,0.4+0.05) \mathrm{Hz}$. The filtered yaw angle $\delta \theta_{f}$ is then given by the Butterworth second order filter as follows:

$$
\delta \theta_{f}=\frac{1.279 s^{2}}{s^{4}+1.599 s^{3}+10.87 s^{2}+7.672+23.01} \delta \theta .
$$

The generator torque input signal is to be preferably low pass filtered. The objective is to ensure a smooth rotor speed, inducing a smooth grid injected generated power. The considered filter is a second order low pass Butterworth, with a cut off frequency of $0.17 \mathrm{~Hz}$ (only frequencies contribution in the rotation). Hence the filtered generator torque is given by:

$$
\delta T_{g f}=\frac{1.141}{s^{2}+1.511 s+1.141} \delta T_{g} .
$$

Figure 9 and Figure 10 show the bode magnitude diagram of the two designed filters. The global scheme of the fault tolerant control strategy is illustrated by Figure 11 Firstly, the tower fore aft (TFA) displacement gradient and rotor speed

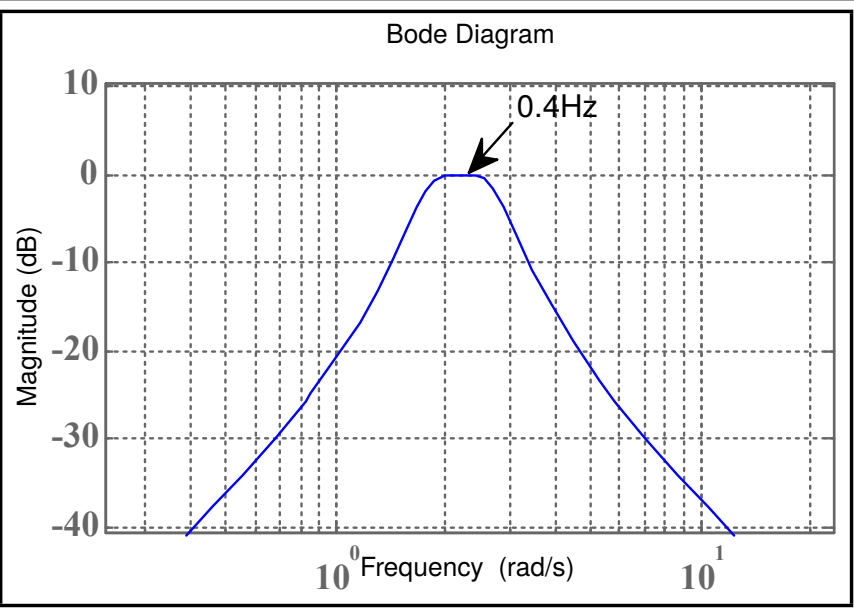

Figure 9. The yaw angle band pass filter magnitude.

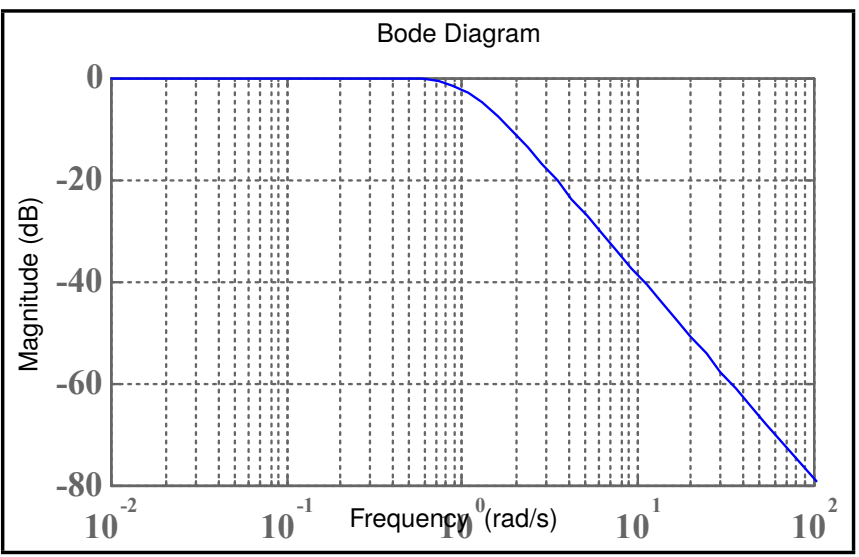

Figure 10. The generator torque low pass filter magnitude (dB).

are measured. The TFA gradient is then integrated to obtain all the measured system states. The states are multiplied by the transition matrix $A$ to constitute the measured dynamics $\dot{Y}_{f}$ (Eq. (19)). Then, the reference dynamics $\dot{Y}_{m}$ (Eq. (20)) are constructed based on the pre-fault pitch control signal $\delta \beta$ which actuates the reference model defined by the pair $\left(A, B_{1}, C\right)$. The residual between $\dot{Y}_{f}$ and $\dot{Y}_{m}$ is then inverted using the pseudo inverse $(C B)^{+}$. The low frequency of the rerouted control signal is directed to the generator as $\delta T_{g}$. The yaw rate control signal $\delta \dot{\theta}$ is integrated to give the yaw angle $\delta \theta$ and then band passed around the tower natural frequency.

\subsubsection{The results of fault tolerant control}

The results of the fault tolerant control strategy are depicted in the figures from Fig. 12 to Fig. 17.

a. The outputs: The rotor speed and the tower fore aft displacement

Figure 12 and Figure 13 illustrate the rotor speed and the tower fore aft vibrations in three case. In the open loop situation, where no control action is made, the wind drives completely the two states to important values far from the operating point.

In the $L Q R$ regulated situation, the rotor speed and the tower fore aft are perfectly regulated about the operating values of 40 rpm and 0.58 meters. 


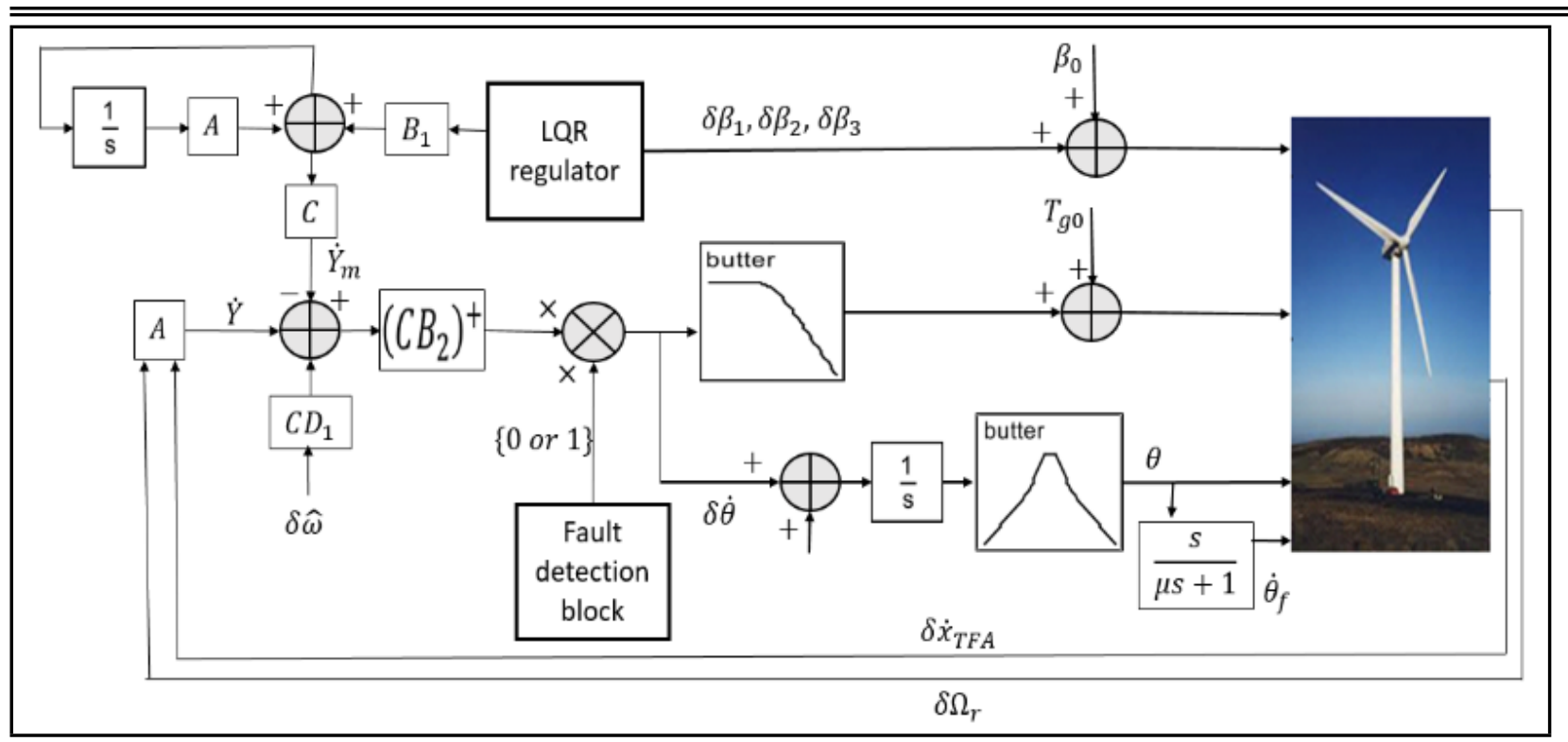

Figure 11. The integrated fault tolerant scheme strategy.

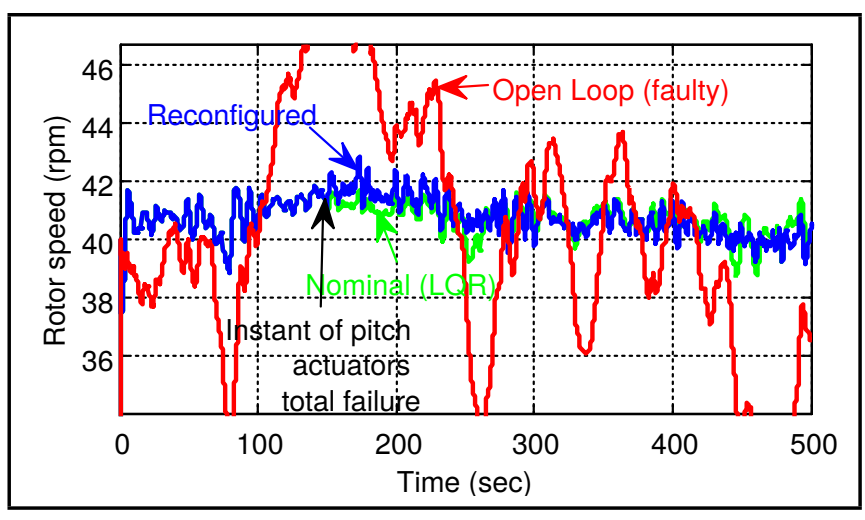

Figure 12. The reconfigured rotor speed compared to the nominal and the faulty situations.

The faulty situation is open loop like due to the complete failure of the pitch actuators. After applying the filtered inverted dynamics strategy, the rotor speed and the tower fore aft dynamics has been perfectly reconstructed.

After the filtering, one can state that the low frequency of the tower fore aft has changed between the nominal LQR and the reconfigured situation. Before the filtering the multi-objective control induces a correlation phenomenon between the low and high frequencies. As a result, the low frequency is passed to the TFA movements. Moreover, the low frequency of the TFA movements is equal (in shape and form only) to the low frequency of the rotor speed. After decorrelation using the proposed strategy, the shape of the low frequency of the TFA becomes independent of the low frequency of the rotor speed

Figure 13 illustrates the Power/Frequency of the tower fore aft in the three situations. The natural frequency of the tower was canceled using the nominal pitch angle used in the LQR regulation. In the faulty situation, the Power/Frequency value of the tower becomes maximal at $0.4 \mathrm{~Hz}$. With the fault tolerant strategy, the resonance frequency canceling capability was perfectly reconstructed.

b. The input signals: the yaw angle and the generator torque

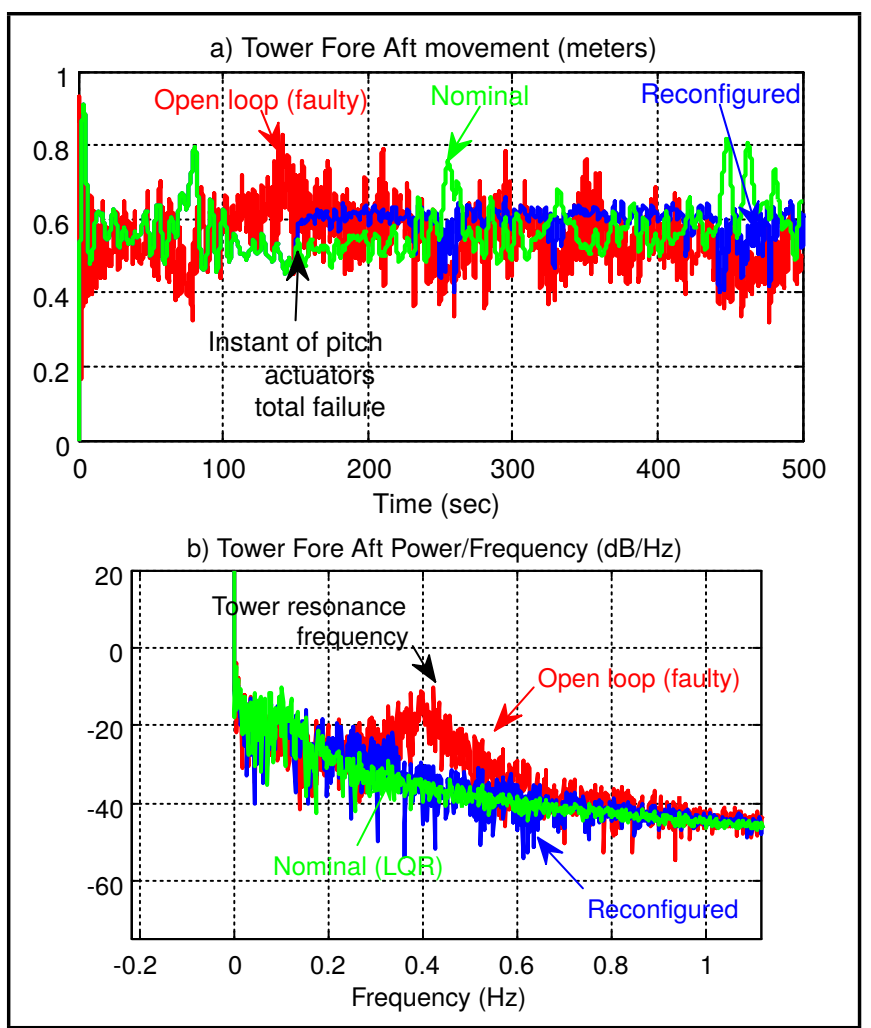

Figure 13. The tower fore aft displacement reconfiguration (a) and the frequency content (b) compared to nominal and faulty situation.

Figure 14 and Fig. 15 illustrates the input signals and their power/Frequency representation in $\mathrm{dB} / \mathrm{Hz}$. Figure $14 \mathrm{c}$ illustrates the yaw angle used in the fault tolerant control. It can be shown that only the high frequencies about $0^{\circ}$ are contained in the yaw angle due to the band pass filter. The needed interval for the control signal was $[-10 ; 10]$ degrees. Figure 14 a confirms the previous remarks by showing a maximal yaw angle magnitude about $0.4 \mathrm{~Hz}$. From the of Figure 14b, between the instants $447 \mathrm{~s}$ and $448 \mathrm{~s}$, the yaw control signal has been repeated twice, which gives a period of $2.7 \mathrm{~s}$; so a frequency of $0.38 \mathrm{~Hz}$ matching the tower natural frequency.

Figure 15a illustrates the generator torque control signal 


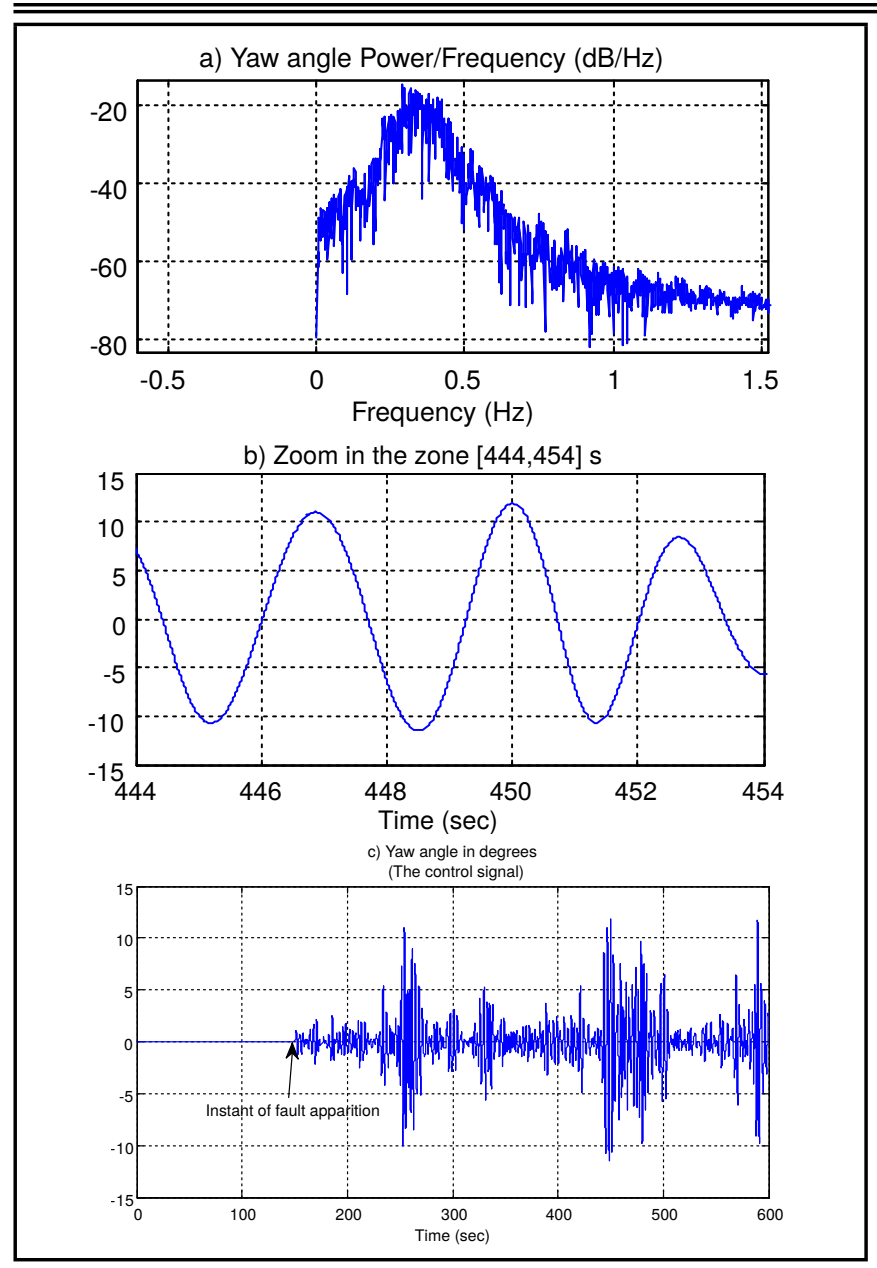

Figure 14. The yaw angle control signal (c), the frequency content (a) and zoom on the region $[444,454] \mathrm{s}(\mathrm{b})$.

used for the rotor speed regulation reconfiguration. The Fig. 15b illustrates the result of low pass filtering of the generator torque. This is manifested as a diminution of the frequencies superior to $0.17 \mathrm{~Hz}$.

Figure 16 illustrates a zoom of the rotor speed and the generator torque in the region [140,200] sec. In Figure 16a, a delta rotor speed of $10 \mathrm{rpm}$ between the operating point and the faulty point has necessitated a control signal of $4000 \mathrm{Nm}$ of generator torque. In fact, the input action from the generator torque to the rotor speed is of $\frac{-175}{J_{t}}$. The rotor speed is then reduced (due to the negative sign of the input action) to $4000 * \frac{175}{J_{t}} * \frac{30}{\pi}=2 \mathrm{rpm}$ about the operating point $40 \mathrm{rpm}$.

Figure 17 represents a zoom in the region [209,212] sec of the tower fore aft and the yaw angle. From the instant 209 s to $210.5 \mathrm{~s}$ for example, the tower fore aft increases of 0.35 meters in 1 second, the yaw angle increases of 3.5 degrees in $1.5 \mathrm{sec}$. The used yaw rate is then $\frac{3.5}{1.5}=2.33 / \mathrm{s}$. Since the natural frequency of the tower is constant, the used yaw rate will be the same over the duration simulation. The value of $2.33 / \mathrm{s}$ matches the existing yaw technology limits.

\subsubsection{Strategy performance evaluation with existing industrial yaw actuator limits}

In the actual existing industrial yaw actuators, some limits on the yaw rate and yaw misalignment are imposed. ${ }^{6,7}$ Namely, an interval of \pm 8 is tolerated on the yaw misalign-

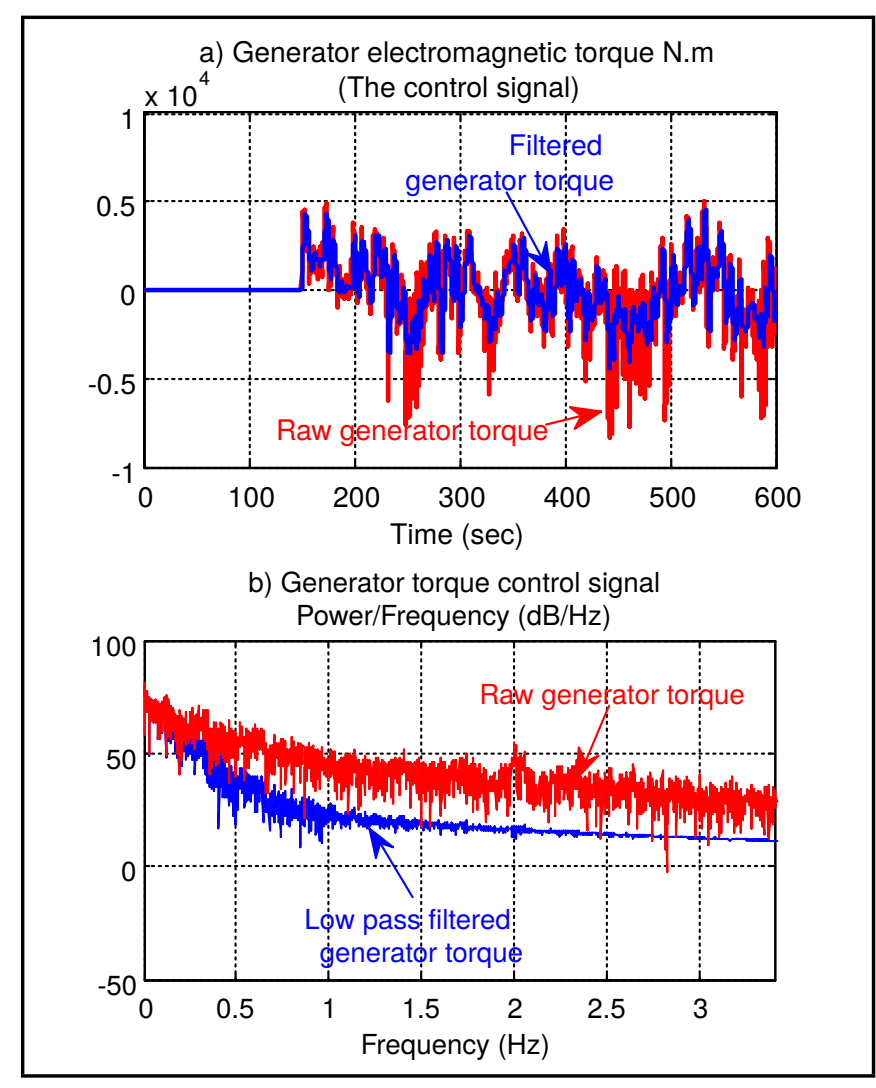

Figure 15. The generator electromagnetic torque control signal (a) and its frequency content (b).

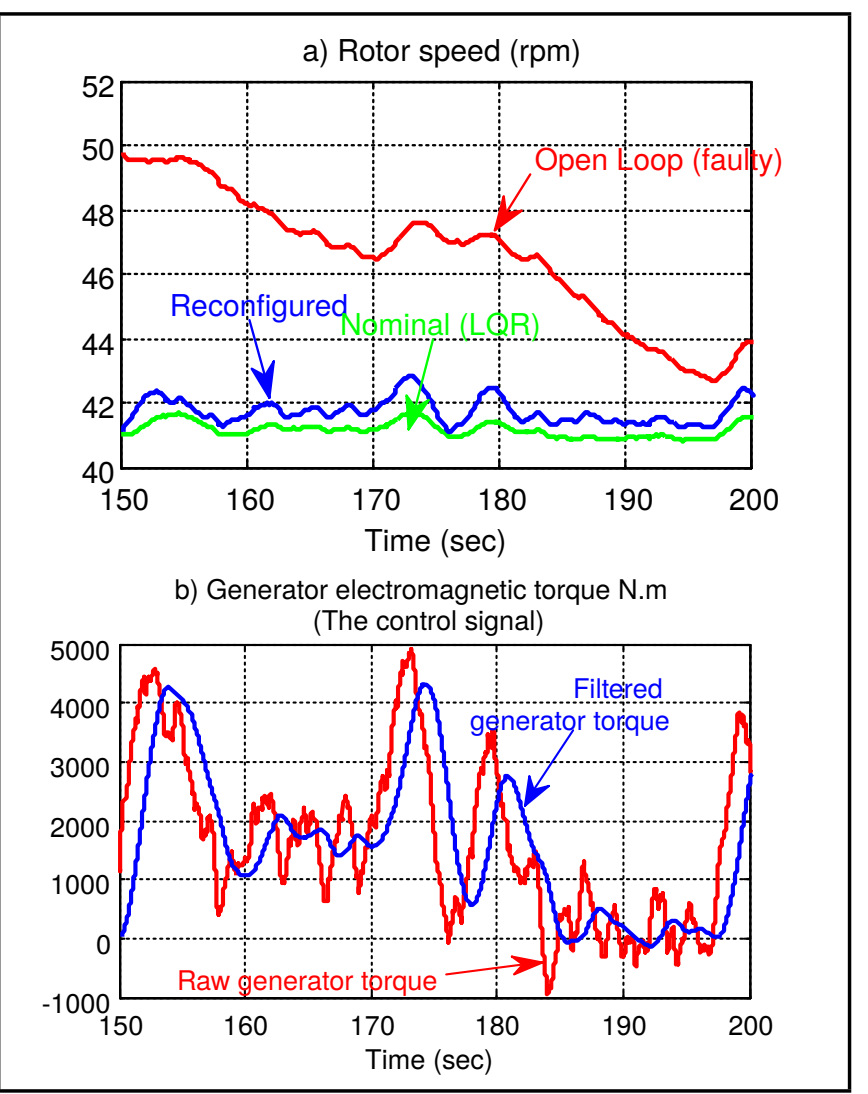

Figure 16. Zoom on the rotor speed (a) and the generator torque (b) in the zone $[150,200] \mathrm{s}$. 


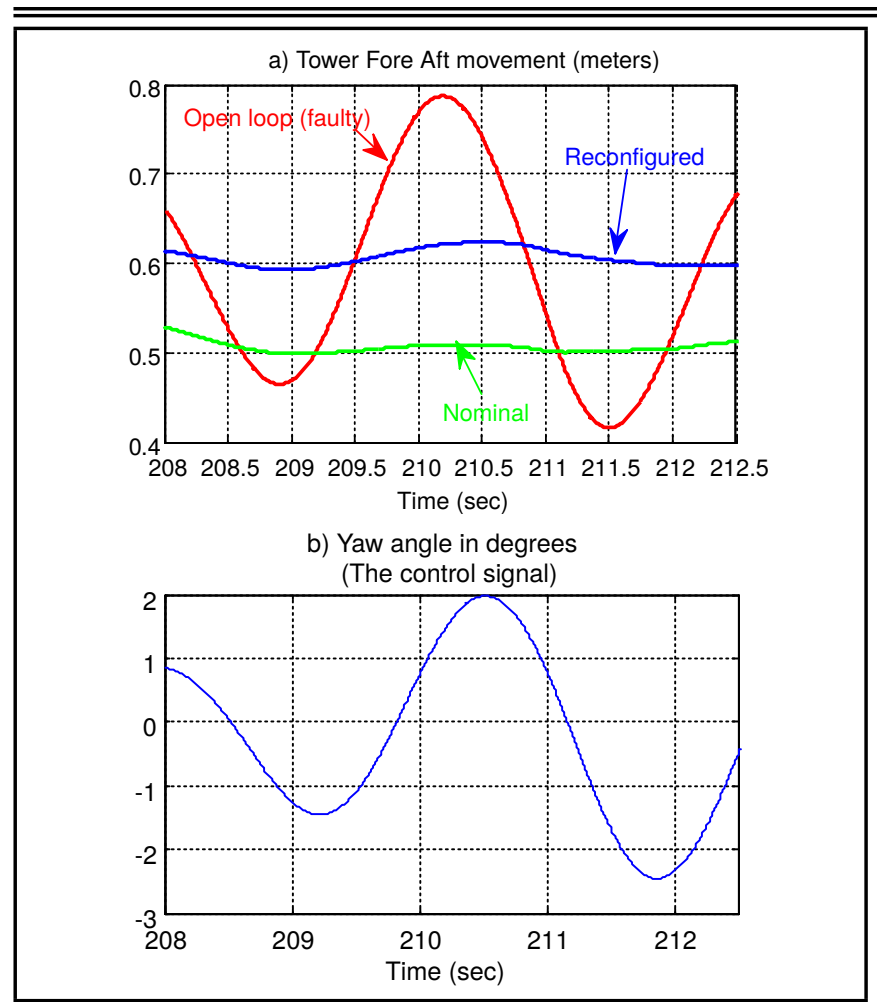

Figure 17. Zoom on the tower fore aft (a) and the yaw rate control signal (b) in the zone $[208.5,212.5] \mathrm{s}$

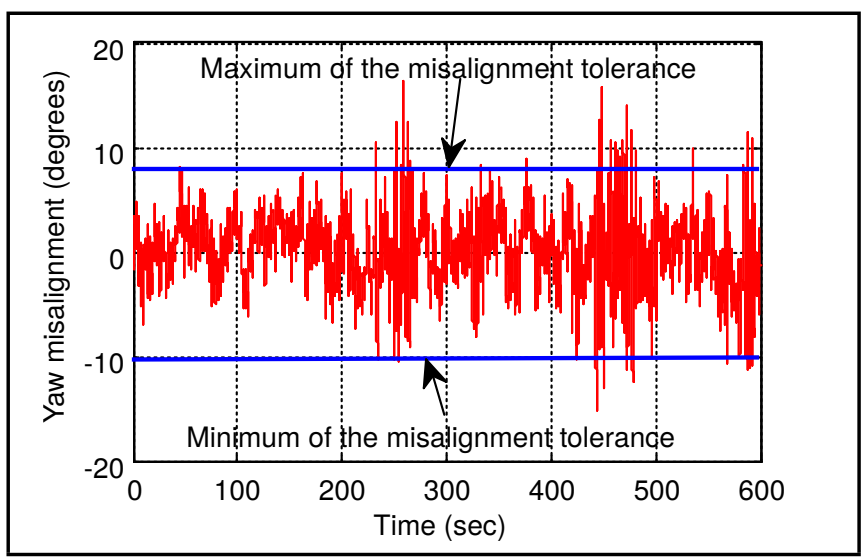

Figure 18. The Yaw misalignment.

ment with a mean value of $0.5^{\circ}$. The industrial yaw rate limits are $\pm 10 / s$, the obtained results fulfil in this interval.

Figure 18 illustrates the created yaw misalignment with the proposed strategy. It is remarkable that the resulting yaw misalignment is contained the most of time in the range of $[-8 ; 8]$ degrees; which belongs to the industrial limit of $[-8,8]$ degrees. Moreover, the mean of the produced yaw misalignment over the $600 \mathrm{~s}$ of simulation is of $0.17^{\circ}$, which means that the rotor speed, and then the power production is not altered in the duration $600 \mathrm{~s}$.

\section{CONCLUSION}

In this paper, a method to control the wind turbine in the presence of total pitch actuator is proposed. Firstly, a residual between the measured dynamics and the wind driven model reference dynamics is proposed. A fault exists when the measured dynamics matches completely the case of the model ref- erence driven only by the wind turbulence. The proposed residual is significant because the fault decision threshold is easily defined as the baseline damping performance. Then the fault tolerant control is based on the redistribution of the reference control signal on the generator and the nacelle yaw actuator. This was performed using the inversion of the new input matrix applied on the residual between the measured dynamics and the reference dynamics. The redistributed signal in the direction of the yaw nacelle was band pass filtered to minimize the yaw misalignment. The choice of the tower natural frequency as the band pass center comes to concentrate the yaw reconfiguration effort on the tower. The generator electromagnetic torque was low filtered to allow a stable energy production. The strategy resulting yaw rate of $9 \%$ is contained in the industrial permitted range of $10 \%$. The used nacelle angle range of $[-5 ; 5]$ degrees remains in the accepted industrial yaw angles of $[-8,8]$ degrees due to the band pass filtering of the yaw angle.

The significance of the paper resides in the fact that the considered fault, the detection method and the reconfiguration strategy were never been considered before in the field. The strategy helps decreasing the stop time of the wind turbines and then the energy production cost.

As perspectives, the case when the generator fails in parallel with the pitch actuators should be considered. In this case, should be studied the capability of the yaw actuator to reconfigure alone and at the same time the rotating movement (rotor speed) and the non-rotating movement (tower vibration). Another study to be performed is the reconfiguration of the blades deflections known by their very high frequency. The capability of the yaw actuator to reconfigure the high frequencies remains to study.

\section{REFERENCES}

1 Cutululis, N. A., and Ceanga, E. Robust control of an autonomous wind power system, Control Engineering and applied informatics, 6 (4), 3-12, (2004).

2 Van Solingen, E., and Wingerden, J. W. van. Linear individual pitch control design for two-bladed wind turbines, Wind Energy, 18 (4), 677-697, (2015). https://dx.doi.org/10.1002/we.1720

3 Bossanyi, E. A. Wind Turbine Control for Load Reduction, Wind Energy, 6 (3), 229-244, (2003). https://dx.doi.org/10.1002/we.95

4 Ors, M. Maximum Power Point Tracking for Small Scale Wind Turbine with Self-Excited Induction Generator, Control Engineering and applied informatics, 11 (2), 30-34, (2009).

5 Vlad, C., Munteanu, I., Bratcu, A.I., and Ceangǎ, E. Anticipative Control of Low-Power Wind Energy Conversion Systems for Optimal Power Regime, Control Engineering and applied informatics, 11 (4), 26-35, (2009).

6 Kim, M. G., and Dalhoff, P. H. Yaw Systems for wind turbines - Overview of concepts, current 
challenges and design methods, Journal of Physics: Conference Series, 524 (1), IOP Publishing, (2014). https://dx.doi.org/10.1088/1742-6596/524/1/012086

7 Kim, M. G., and Dalhoff, P. H. Investigation of yaw system loads for a wind turbine in different operation and load cases, 2nd Conference for Wind Power Drives CWD, Aachen University, (2015).

8 Chen, J., and Patton, R. J. Robust Model-Based Fault Diagnosis for Dynamic Systems, (1999). https://dx.doi.org/10.1002/rnc.615

9 Kou, Q., Sun, D. H., Li, Z. X, Qiao, S. J., Hou, Y.J., and Shi, Y.T. $\mathrm{H}_{\infty}$ Fault Tolerant Control of Wind Turbine System with Actuator Faults, IFAC Proceedings Volumes, 47 (3), 5838-5843, 2014. https://dx.doi.org/10.3182/20140824-6ZA-1003.01244

${ }^{10}$ Elkhatib, K., Aitouche, A., and Bayart, M. Fault Tolerant Control of Wind Energy Conversion Systems Subject to Sensor Faults, Sustainability in Energy and Buildings. Smart Innovation, Systems and Technologies, 12. Springer, Berlin, Heidelberg, (2012). https://dx.doi.org/10.1007/9783-642-27509-8_42

11 Precup, R. E., Angelov, P., Costa, B. S. J., and Mouchaweh, M. S. An overview on fault diagnosis and nature-inspired optimal control of industrial process applications, Computers in Industry, 47, 75-94, (2015). https://dx.doi.org/10.1016/j.compind.2015.03.001

12 Ribrant, J., and Bertling, L. M. Survey of failures in wind power systems with focus on Swedish wind power plants during 1997-2005, IEEE Transactions on Energy Conversion, 22 (1), 167-173. (2007). https://dx.doi.org/10.1109/PES.2007.386112

${ }^{13}$ Elmaati, Y. A., Bahir, L. El, and Faitah, K. Fault Tolerant Control of Internal Faults in Wind Turbine: Case Study of Gearbox Efficiency Decrease, International Journal of Rotating Machinery, 2018, 1-8, (2018). https://dx.doi.org/10.1155/2018/9538489

${ }^{14}$ Elmaati, Y. A., Bahir, L. El, and Faitah, K. An integrator based wind speed estimator for wind turbine control, Wind and structures, 21 (4), 443-460, (2015). https://dx.doi.org/10.12989/was.2015.21.4.443

15 Rezaei, V., and Johnson, K. E. Robust Fault Tolerant Pitch Control of Wind Turbines, 52nd IEEE Conference on Decision and Control, Florence, December, (2013). https://dx.doi.org/10.1109/CDC.2013.6759913

${ }^{16}$ Rezaei, V., and Salmasi, F. R. Robust Adaptive Fault Tolerant Pitch Control of Wind Turbines, Wind Engineering, 38 (6), 601-612, (2011). https://dx.doi.org/10.1260/0309$524 X .38 .6 .601$
17 Kühne, P., Pöschke, F., Schulte, and F. Fault estimation and fault-tolerant control of the FAST NREL 5MW reference wind turbine using a proportional multiintegral observer, International Journal of Adaptive Control and Signal Processing, 32 (4), 568-585, (2018). https://dx.doi.org/10.1002/acs.2800

${ }^{18}$ Lan, J., Patton, R.J., and Zhu, X. Fault-tolerant wind turbine pitch control using adaptive sliding mode estimation, $R e$ newable Energy, 116 (Part B, February), 219-231, (2018). https://dx.doi.org/10.1016/j.renene.2016.12.005

${ }^{19}$ Shi, Y.T., Xiang, X., Wang, L., Zhang, Y., and Sun, D.H. Stochastic Model Predictive Fault Tolerant Control Based on Conditional Value at Risk for Wind Energy Conversion System, Energies, 11 (1), 193, (2018). https://dx.doi.org/10.3390/en11010193

${ }^{20}$ Wei, X., and Verhaegen, M. Sensor and actuator fault diagnosis for wind turbine systems by using robust observer and filter, Wind Energy, 14 (4), 491-516, (2014). https://dx.doi.org/10.1002/we.438

21 Jonkman, J. M., and Buhl, M. L. Fast user's guide, (2005). National Renewable Energy Laboratory, https: //nwtc.nrel.gov/FAST7, (accessed 10 June 2018).

22 Jonkman, J. M., and Buhl, M. L. TurbSim user's guide, (2007). National Renewable Energy Laboratory, https://nwtc.nrel.gov/TurbSim, (accessed 10 June 2018).

23 Jong-Won, L., Joong-Kwan, K., Jae-Hung, H., and HyungKee, S. Active load control for wind turbine blades using trailing edge flap, Wind and Structures, 16 (3), 263-278, (2013). https://dx.doi.org/10.12989/was.2013.16.3.263

${ }^{24}$ Poore, R., and Lettenmaier, T. Alternative Design Study Report: WindPACT Advanced Wind Turbine Drive Train Designs Study, Global Energy Concepts, LLC Kirkland, Washington, (2002). https://dx.doi.org/10.2172/15004456

25 Perfiliev, D., Hämäläinen, J., and Backman, J. Robust Analyzing Tools for Wind Turbine Blades Coupled with Multiobjective Optimization, Journal of Energy and Power Engineering, 7, 1831-1836, (2013).

${ }^{26}$ Shamsuzzoha, M., and Skogestad, S. The setpoint overshoot method: A simple and fast closed-loop approach for PID tuning, Journal of Process Control, 20 (10), 1220-1234, (2010). https://dx.doi.org/10.1016/j.jprocont.2010.08.003

27 Cho, S., Gao, Z., and Moan, T., Model-based fault detection, fault isolation and fault-tolerant control of a blade pitch system in floating wind turbines, Renewable Energy, 120 (May), 306-321, (2018). https://dx.doi.org/10.1016/j.renene.2017.12.102 